\title{
Puerto Rico: los dilemas del status político y el desarrollo dependiente
}

\section{INTRODUGGIÓN}

Puerto Rico representa un caso excepcional en la historia de América Latina debido a la naturaleza de sus relaciones con Ios Estados Unidos. Si bien es cierto que el Continente en su conjunto inauguró el siglo veinte con los Estados Unidos jugando el papel predominante antes detentado por potencias europeas tales como las de España e Inglaterra, Puerto Rico representa el único caso en que Norteamérica no sólo interviene militarmente en la Isla, sino que mantiene su presencia en ella aún hasta el presente. Sin embargo, el status de Puerto Rico es ambiguo, y no resulta claro en qué medida, como muchos críticos sugieren, es la Isla una colonia de los Estados Unidos. También es cierto que Puerto Rico no es de ninguna manera un país independiente, dado que su territorio pertenece a los Estados Unidos, y que su importancia económica y estratégica se encuentra definida por este país. Puerto Rico, en un sentido general, es una dependencia norteamericana, pero con una medida de autonomía política local que lo aparta de la definición estricta de colonia.

La falta de precisión en torno al status de Puerto Rico guarda estrecha relación con el desarrollo económico del país. Sin la ambigüiedad en lo político, el actual sistema económico no habría podido sostenerse, dado que requiere, para su funcionamiento, de una peculiar relación entre Estados Unidos y Puerto Rico. Esta Isla ha experimentado un desarrollo económico espectacular en las últimas cuatro décadas debido a su asociación con los Estados Unidos. Los más optimistas sugieren que es tal el éxito del modelo, que debiera extenderse al resto de América Latina. Puerto Rico goza, de hecho, del mejor ingreso per cápita en el Continente, aunque éste sea todavía bastante inferior al de los Estados Unidos. Sin embargo, las crisis que aquejan a este último país afectan doblemente a una dependencia como la de Puerto Rico. Así, hay más mérito en examinar el desarrollo de la Isla no tanto en comparación con el resto de América Latina, sino a la luz, sobre todo en el siglo veinte, de 
su relación con los Estados Unidos. Este desarrollo, particularmente en lo económico, se encuentra íntimamente ligado al problema del status político. Una cosa conlleva la otra, y da lugar a los dilemas de los cuales se ocupará este estudio en las siguientes páginas.

\section{Puerto Rico bajo LA DOMINAGión ESPAÑola}

Puerto Rico tiene una historia común con América Latina durante todo el período de la dominación colonial española. Sin embargo, hay algunos antecedentes que apartan a la Isla del resto de las colonias desde un comienzo mismo. Puerto Rico parece haber sido desde un principio la víctima de fuerzas que jamás pudo controlar. El hecho mismo de su colonización: lleva el sello de las disputas entre la Corona española y la dinastía Colón. De acuerdo a las Capitulaciones de Santa Fe, firmadas por Cristóbal Colón y la Corona en 1492, todos los territorios descubiertos o por descubrir caían bajo la jurisdicción del Almirante. Sin embargo, sus dificultades como gobernante en la Isla Hispaniola (hoy República Dominicana y Haití) Ie impidieron colonizar Puerto Rico con prontitud. Y mientras el hijo de Golón, Diego, luchaba en la Corte por los derechos de su padre ya fallecido, Ia Corona no perdió el tiempo en enviar sus propios emisarios para colonizar la Isla. Juan Ponce de León, con autorización del Rey, funda Puerto Rico y establece los primeros pactos con los indios en 1508. Ponce, sin embargo, no tuvo mayor tiempo de llevar a cabo su obra colonizadora. En 1509, Diego Colón gana el litigio en contra de la Corona por sus derechos, y viola los acuerdos que restringen su autoridad sobre Puerto Rico al enviar sus propios delegados por sobre la autoridad del Rey. La importancia de estas disputas trasciende el mero carácter legal. El asunto del poder en el Caribe y en las colonias españolas del siglo dieciséis estaba intimamente ligado con la posesión de la tierra y la distribución de indios como fuerza de trabajo bajo la institución denominada encomienda. Cualquier cambio de poder tenía efectos inmediatos sobre la distribución de la fuerza de trabajo, y por ende, sobre la economía de la Isla. En el caso de Puerto Rico, los cambios frecuentes en la propiedad de la encomienda originados por los problemas de poder llevaron a la rebelión indígena de 1511. Cuando se compara esta rebelión con otra similar en Hispaniola, vemos que en esta última la rebelión tomó muchos más años que en Puerto Rico. La rapidez de esta última se explica por los cambios acelerados en el sistema de encomienda y los problemas de poder en el Caribe, de los cuales Puerto Rico fue una de las primeras víctimas. La rebelión de 1511 tuvo efectos desastrosos para la población indígena de la isla. La mayoría fue eliminada durante el enfrentamiento con los españoles o en la persecución posterior. Para 1520, prácticamente no había indios en Puerto Rico. Es bien sabi- 
do que los españoles contaban con la mano de obra indígena para Ia explotación de las minas, de modo que tanto el agotamiento del oro en esta isla, como el exterminio de la población indígena, trajeron graves problemas para España, que se vio enfrentada ante la posibilidad de tener que abandonar su dominio sobre el territorio. Sin embargo, la importancia estratégica de la isla, debida a su ubicación privilegiada en el Caribe, impulsó a las autoridades a mantener esta colonia a toda costa, aún a pesar de los inconvenientes económicos que esto originaba. A esta altura, ya los imperios Inca y Azteca habían sido conquistados, de modo que el grueso de las riquezas extraídas de tales territorios debían pasar por el Caribe con rumbo a España. Para la Corona resultaba vital tener bajo su control aquellas islas que facilitaran el tráfico de riquezas en el Caribe, y defendieran las rutas de posibles ataques ${ }^{\mathbf{1}}$.

Las aprehensiones de la Corona a este respecto eran justificadas. Los primeros ataques franceses a Puerto Rico datan de la década de los treinta en el siglo dieciséis. Tales ataques tenían el propósito, por una parte, de erosionar el poder de España en la región, y al mismo tiempo controlar las rutas comerciales hacia y desde España. De la misma fecha datan las fortificaciones que aún hoy día adornan la isla, fortificaciones que la hicieron prácticamente inexpugnable durante siglos, con la excepción de las breves invasiones británicas y holandesas en 1598 y 1625 , respectivamente. El costo de tal mantenimiento, sin embargo, resultaba bastante alto, pero vital para las necesidades del Imperio. Aún así, resultó prácticamente imposible para España mantener las potencias europeas ta-

${ }^{1}$ La bibliografía sobre el Caribe y Puerto Rico en el siglo XYI es demasiado extensa para citarla en su totalidad. Entre las fuentes más importantes, sin embargo, se encuentran las siguientes: Para el perfodo pre-colombino, José Juan Arróm, Mitología y artes prehispánicas de las Antillas (México: Siglo xxu Editores, 1975); Ramón Pané, Relación acerca de las antiguiedades de los indios, ed. por J. J. Arróm México: Siglo xxı Editores, 1976) Mercedes López-Baralt, El mito taino: Raiz y proyecciones en la amazonia continental (Puerto Rico: Ediciones Huracán, 1976) y Francisco Moscoso, "Chiefdom and Encomienda in Puerto Rico: The Development of Tribal Society and the Spanish Colonization to 1530", en AdaIberto López, ed., The Puerto Ricans: Their History, Culture and Society, (Cambridge, Mass.: Schenkman Publishing Co., 1980) Sobre el descubrimiento, colonización y conquista, A. M. Salas y A. R. Vázquez, Noticias de la Tierra Nueva, (Buenos Aires: Editorial Universitaria de Buenos Aires, 1964); Charles Gibson, Spain in America (New York: Harper and Row, Publishers, 1966) y J. F. Parry y Philip Sherlock, Historia de las Antillas, trad. por Viviana S. de Ghio (Buenos Aires: Editorial Kapelusz, S. A., 1976). Sobre la conquista y colonización de Puerto Rico, en particular, Salvador Brau, La colonización de Puerto Rico (San Juan: Instituto de Cultura Puertorriqueña, 1966); Manuel Ballesteros Gaibrois, La idea colonial de Ponce de León: Un ensayo de interpretación (San Juan: Instituto de Gultura Puertorriqueña, 1960); Eugenio Fernández Méndez, Proceso histórico de la conquista de Puerto Rico (San Juan: Instituto de Cultura Puertorriqueña, 1970) y Loida Figueroa, History of Puerto Rico (New York: Anaya Books Co., 1972). 
les como Francia, Inglaterra y Holanda fuera del Caribe. No sólo sufre sus ataques frecuentes, sino que tales países comienzan a ocupar aquellas islas antillanas que España no tuvo oportunidad de colonizar. Desde tales islas, estas potencias no sólo presentaron graves problemas para las pretensiones hegemónicas de España sino que también iniciaron un tipo de economia que habría de cambiar la faz de la región: el sistema de plantaciones ${ }^{2}$.

Mientras España mantenía Puerto Rico, Cuba e Hispaniola como bastiones militares para defender sus rutas comerciales y proveer abastecimientos a sus flotas, las otras potencias europeas introdujeron el monocultivo del azúcar. Este cultivo trajo consigo la esclavitud africana en grande escala, y un tipo de economía que serviría de base a un nuevo orden económico mundial: la explotación comercial de la agricultura, la venta de sus productos en un mercado internacional, y la acumulación de capital en los grandes centros europeos. El Caribe pasa, así, a conformar la periferia productora de los centros comerciales instalados en Europa.

Los intereses de España eran totalmente distintos a los de las otras potencias europeas. Para la Corona española, inicialmente, el cultivo del azúcar no resultaba tan importante como la explotación del oro y la plata. $\mathrm{O}$ al menos, no manifestó mayor interés por la producción agrícola mientras hubiera minerales que extraer en aquellas regiones con abundante mano de obra indígena. Islas como Puerto Rico tenían un lugar aún más secundario dentro del esquema imperial español: un mero papel estratégico y militar ${ }^{3}$.

Por estas razones, y también por las frecuentes amenazas provenientes de las islas vecinas, la economía de Puerto Rico fue, durante la mayor parte de la dominación española, totalmente dependiente. Incapaz de producir lo necesario para su consumo interno, la isla debía sobrevivir con Ios subsidios que la Corona le otorgaba a través del Virreinato de Nueva España, y que se conocen con el nombre de Situado Mexicano. Sólo muy tarde, en el siglo diecinueve, cuando el sistema de plantaciones empezaba a decaer en las otras islas, Puerto Rico comienza a producir azúcar. Como en las otras islas, las plantaciones de azúcar requieren mano de obra esclava. Aunque nunca en la proporción de las colonias francesas y británicas, Puerto Rico tuvo, durante el período de las plantaciones, su

\footnotetext{
${ }^{2}$ Respecto a la entrada de otras potencias europeas en el Caribe puede consultarse el libro ya citado de Parry. y Sherlock, $Y$ la segunda sección del libro de Roberta M. Delson, Readings in Garibbean Fiistory and Economics: An Introduction to the Region (New York, London, Paris: Gordon and Breach Science Publishers, 1981).

"Adalberto López, "The Beginnings of Colonization: Puerto Rico, 1493-1800", en James Petras and Adalberto López eds. Puerto Rico and Puerto Ricans: Studies in History and Society (New York: Schenkman Publishing Co., 1974); pp. 12-41.
} 
número más alto de esclavos. Al mismo tiempo, hubo otras consecuencias sociales derivadas de este tipo de economía. La Corona española, incapaz de impedir la instalación de fugitivos tanto españoles como extranjeros provenientes de otras islas en el interior deI país, había permitido sin quererlo el florecimiento de un estilo de economía basada en la producción agrícola de subsistencia. Con el afán de desarrollar económicamente la isla, la Corona concentró sus esfuerzos en reclamar estas tierras y entregarlas a la producción de azúcar, mediante leyes promulgadas en 1815 y 1824. Así, durante el siglo diecinueve, Ios campesinos se vieron obligados a servir como mano de obra asalariada en las plantaciones, mientras que la economía basada en la producción agrícola de subsistencia comenzó a desaparecer. A partir de entonces, la agricultura de este tipo habría de sufrir un golpe tras otro, dada la urgencia española de mantener una economía capaz de producir la mayor cantidad de beneficios en la menor cantidad de tiempot.

Uno de los mayores problemas del sistema económico basado en la comercialización de la agricultura radica en la vuInerabilidad del producto que se cultive respecto a las fluctuaciones del mercado mundial. De modo que cuando la superproducción de azúcar disminuyó drásticamente los precios de este producto, la economía de Puerto Rico, como la de otros países en una situación similar, se vio seriamente afectada. Durante el siglo diecinueve, entonces, se pasó de la producción de azúcar a la de café, y de la de café a la de tabaco. En cada una de estas instancias, sin embargo, el patrón es el mismo, de manera que la economía de la isla depende fundamentalmente de las fluctuaciones de precios en el mercado mundial.

Este tipo de vulnerabilidad dio curso a una de las pocas rebeliones significativas en la historia del siglo diecinueve en Puerto Rico. Las divisiones sociales generadas por el sistema de plantaciones precipitaron un conflicto entre los productores y los comerciantes de café en el interior de la isla en 1868. Los últimos tenían el apoyo de la Corona, en tanto que los productores se encontraban a merced de las fluctuaciones del mercado. Éstos, apoyados por los peones y algunos esclavos, tomaron brevemente la ciudad de Lares en ese año y proclamaron la República Independiente de Puerto Rico. La fuerza militar española en la isla, sin embargo, se había visto incrementada debido a la pérdida de sus colonias en el

'En cuanto a Ia esclavitud en Puerto Rico, puede consultarse la obra clásica de Luis Dfaz Soler, La esclavitud negra en Puerto Rico (Rfo Piedras: Editorial Universitaria, 1965). En cuanto al cultivo del azúcar y su relación con la esclavitud, Sidney Mintz, "Labor and Sugar in Puerto Rico and in Jamaica, 18001850", en Laura Foner and Eugene D. Genovese, eds., Slavery in the New World: A Reader in Comparative History (Englewood Cliffs, N. J.: Prentice Hall, Inc., 1969), pp. 170-177. Para una visión de conjunto sobre el perfodo, Adalberto López, "Socio-Politico Developments in a Colonial Context: Puerto Rico in the Nineteenth Century", en Petras y López, Puerto Rico, pp. 43-86. 
resto de América Latina. Ast, la rebelión de Lares fue aplastada con relativa facilidad. Aunque el conflicto tuvo un carácter antiespañol por momentos, y manifestó un cierto grado de descontento respecto a las políticas de la Corona, este enfrentamiento fue fundamentalmente local, y producto de los problemas inherentes a una economía basada en la agricultura comercial ${ }^{\vec{b}}$.

El Grito de Lares, como se conoce a esta rebelión, se ha transformado en un símbolo nacionalista, y es frecuente verlo descrito como un movimiento que, aunque frustrado, representa un esfuerzo puertorriqueño genuinamente independentista. Sin embargo, es poca la eyidencia que indica que las motivaciones de este conflicto, como las que llevaron a las guerras de independencia en el resto de América Latina, hayan tenido un carácter nacional. Se menciona generalmente a Ramón Emeterio Betances como uno de los líderes de esta rebelión, a pesar de que su papel fue relativamente marginal en el conflicto mismo. Los escritores más nacionalistas, de hecho, tienden a identificar a Betances con la conciencia revolucionaria del momento ${ }^{6}$ Pero la evidencia disponible sugiere que, aunque Betances expresó las aspiraciones de un sector importante del país, el movimiento de Lares fue un suceso aislado, y que sus participantes fracasaron en conseguir un apoyo más generalizado para su movimiento.

Sin embargo, hay una serie de otros factores que explican el fracaso de Lares. España ya había perdido la mayoría de sus colonias latinoamericanas. Por ende, se encontraba en una situación bastante más aventajada para defender estos últimos bastiones (Cuba y Puerto Rico) de sus extensos dominios. En tales condiciones, fue bastante más fácil para España sofocar la rebelión. Al mismo tiempo, la opinión mayoritaria del momento no favorecía tanto la independencia como alguna suerte de negociación con España que permitiera mayor autonomía para la isla. De hecho, el partido más importante de la época se denominaba Partido Autonomista, seguido en popularidad por el Partido Asimilista. Aquellos que favorecían la independencia total eran muy pocos, y no llegaron nunca a constituir una fuerza polftica significativa. También es cierto que Ios independentistas sufrieron una persecución más aguda. Pero su falta de apoyo se explica por la carencia de conexiones con los sectores más amplios de la población. Tanto Ios asimilistas como los

'Laird W. Bergard, "Toward Puerto Rico's Grito de Lares: Coffee, Social Stratification, and Class Conflicts, 1828-1868", Hispanic American Historical R'eview 60 (November 1980), 617-642. También, James W. Wessman, "The Demographic Structure of Slavery in Puerto Rico: Some Aspects of Agrarian Capitalism in the Late Nineteenth Century", Joumal of Latin American Studies 12 $(2,1980), 271-289$.

Especialmente Manuel Maldonado-Denis, Puerto Rico: A Socio-Fistoric Interpretation, trad. por Elena Vialo (New York: Vintage Books, 1972). 
autonomistas pensaban que, dada la situación política y militar de España en el Caribe luego de las guerras latinoamericanas de independencia, hubiera sido práctiçamente suicida iniciar un movimiento de independencia total. Este temor parece haber tenido un efecto devastador en las aspiraciones políticas del momento. Así, la negociación con España tuvo precedencia sobre el independentismo. Betances, por otra parte, aunque no contaba con mayor apoyo, tenía plena razón en sugerir que resultaba bastante ingenuo esperar reformas desde España. De hecho, España otorgó la autonomía política a Puerto Rico sólo cuando se encontraba al borde de perder sus últimas colonias en el Caribe.

\section{LA GUERRA HISPANOAMERICANA DE 1898 Y LOS INIGIOS DE LA INTERVENGIÓN NORTEAMERIGANA EN PUERTo Rico}

La guerra hispanoamiericana de 1898 cambió fundamentalmente la faz del Caribe. En esta guerra, España perdió sus últimas posesiones, Cuba y Puerto Rico, que pasaron a manos de los Estados Unidos, la nueva potencia dominante en la región. Las razones del expansionismo norteamericano radican en su crecimiento industrial, acelerado luego de la Guerra Civil, y la búsqueda de nuevos mercados para su economía industrial. También es cierto que las consideraciones estratégicas, como lo habian sido antes para España y otras potencias europeas, resultaban preponderantes. Las islas de Puerto Rico y Cuba se encuentran en la entrada del Garibe y de la costa sur de Ios Estados Unidos. Pero las consideraciones estratégicas y económicas no eran todas las existente. Había también otras motivaciones, no ya religiosas como las había tenido España para justificar ideológicamente su colonización y conquista, sino esta vez de expansión de las doctrinas democráticas. El conocido "Destino Manifiesto" suministró el credo que justificó, al menos ideológicamente, la expansión norteamericana más allá de sus fronteras naturales. Obviamente, había un diseño estratégico tras la expansión. Una vez consolidada la ocupación en Puerto Rico y Cuba, comienza de hecho la construcción del Canal de Panamá en 19037.

Puerto Rico pasó de manos españolas a manos norteamericanas sin tener influencia alguna en el proceso. Al parecer, las necesidades estratégicas militares y económicas de las grandes potencias mundiales han sido de tal magnitud como para reprimir, controlar o desalentar cualquier iniciativa relacionada con el control local o la independencia de la isla. Puerto Rico, de todas maneras, aceptó la

${ }^{7}$ Gordon Lewis califica este periodo como "el surgimiento del Mediterráneo norteamericano" en su obra Puerto Rico: Freedom and Power in the Caribbean (New York: Monthly Review Press, 1963). La expansión norteamericana también se encuentra descrita por Gabriel Kolko, Main Curvents in Modern American History (New York: Harper and Row, Publishers, 1976), 34-66: 
intervención norteamericana si no con entusiasmo, al menos con grandes expectativas. La situación económica bajo el dominio español era lo suficientemente desastrosa como para esperar algo mejor de cualquier otro tipo de situación política. Cerca del 80 por ciento de la población era analfabeta en el momento de la intervención norteamericana, y su población rural se encontraba diezmada por las enfermedades tropicales.

La intervención norteamericana trajo alivios para estos problemas, pero agravó otros. En primer Iugar, aquellos relativos al status político, y los relativos a la economía. En relación al status poÍ́tico, Puerto Rico pasó a ser parte del territorio de los Estados Unidos sin llegar a formar parte integral de los Estados Unidos. En relación a la economía, Ios Estados Unidos promulgaron una serie de decretos, el más conocido de los cuales es la Ley Foraker de 1900, que dio a los Estados Unidos control absoluto sobre el comercio y la explotación de los recursos de esta isla ${ }^{8}$. El Congreso norteamericano, de hecho, se vio asediado por grupos económicos en buąca de permisos y garantías para operar en Ia isla. De acuerdo a estos intereses, Puerto Rico, que durante las últimas décadas del siglo diecinueve había transformado el café en su producto de exportación más importante, volvió súbitamente a la producción de azúcar; la que predominó en esta isla hasta la década de los treinta en el presente siglo. Tal como en el período español de las plantaciones, bajo la intervención norteamericana sc agudizaron los conflictos inherentes a la economía de monocultivo: las divisiones sociales y la vulnerabilidad de la economía respecto a los cambios de precios en el mercado. La mayoría de los terrenos pasaron a manos de compañías norteamericanas que terminaron destruyendo a los propietarios locales.

La concentración de la tierra en grandes plantaciones controladas por empresas norteamericanas se encontraba sancionada por la Ley Foraker. EI sistema de contribuciones exigía además impuestos sobre la propiedad antes que sobre la producción, de modo que muchos pequeños propietarios, en años de escasez o mala cosecha, se vieron forzados a pagar sus deudas de impuestos con terrenos. Así fueron creciendo las posesiones de las empresas norteamericanas, que eran las únicas con capital disponible en ese momento. Como sugiere un autor, hacia 1930 Puerto Rico se había transformado en una típica isla azucarera ${ }^{9}$.

De esta manera se acentuó la dependencia de la isla respecto al mercado y sus fluctuaciones. La depresión de los años treinta, unida a la crisis del comercio internacional, llevaron a Puerto Rico a

"Un análisis detallado de la Ley Foraker puede encontrarse en Lyman Jay Gould, "The Foraker Act: The Roots of American Colonial Policy" (Ph. D. dissertation, University of Michigan, 1958).

${ }^{8}$ Lewis, p. 92. 
una situación de gravedad extrema, no sólo en lo económico, sino también en lo político. Durante los primeros años de la intervención, los Estados Unidos, a través de la Ley Foraker, mantenían el control político de la isla mediante un Gobernador y un Comité Ejecutivo designados por el- Presidente de los Estados Unidos. Si bien es cierto que cinco de los once miembros de este Comité eran puertorriqueños, todos ellos eran nombrados por el Presidente nornorteamericano. Con la Ley Jones de 1917, varias funciones del Comité fueron entregadas a un aparato legislativo elegido popularmente en Puerto Rico, aunque sus poderes estaban sujetos al veto presidencial. Gon esta ley, además, los puertorriqueños recibieron ciudadanía norteamericana. Esta última franquicia, aparte de no otorgar ni derecho a voto ni representación ante el Congreso de los Estados Unidos, limitaba la autonomía política de Puerto Rico y sus posibilidades de independencia.

Así, durante las primeras décadas de la intervención norteamericana, los líderes locales buscaron diferentes fórmulas de acomodo dentro del nuevo sistema, al entender que la intervención norteamericana adquiría un carácter cada vez más permanente. Tal como en el siglo anterior en relación a España, había quienes planteaban la incorporación completa a la metrópolis, los que exigían algún grado de autonomfa, y quienes reclamaban la independencia completa. Lo mismo ocurría en relación a la dominación norteamericana, sólo que la agudización de los problemas económicos durante la década de los treinta, junto a la falta de una definición clara respecto al status político de la isla, condujo a un grado mucho más alto de militancia independentista. Al Betances del siglo diecinueve corresponde en este período Pedro Albizu Campos y el recientemente creado Partido Nacionalista. La desilusión respecto a los logros de la intervención norteamericana llevaron a muchos a plantear la independencia como única solución posible para Puerto Rico, incluidos muchos miembros del Partido Liberal, de tendencia más moderada. Para algunos independentistas, inspirados tal. vez en la figura de Betances, resultaba muy poco probable que se consiguieran mayores concesiones de parte de los norteamericanos, quienes hasta ese momento seguían designando sus gobernadores sin participación puertorriqueña. Los habitantes de la isla sólo podían votar por los miembros de las 'Cámaras Legislativas, mientras que el Poder Ejecutivo permanecia en manos de Washington. Los nacionalistas, desilusionados en los años treinta por los procedimientos de la metrópolis, además de impulsados por su fracaso en el intento electoral del año 1932, procedieron a un cambio de tácticas que precipitaron una crisis política de magnitud.

En 1936, el Jefe de Policía, Francis Riggs, fue asesinado por miembros de la Juventud Nacionalista, luego que algunos de sus propios miembros fueron brutalmente reprimidos por la policla en 
la Universidad de Puerto Rico. Inmediatamente a continuación, nuevas protestas condujeron a mayor represión, llegando ésta a un punto cúlmine con la llamada "Masacre de Ponce", donde varios manifestantes fueron muertos o heridos. Con el propósito de destruir el movimiento, las autoridades encarcelaron a Pedro Albizu Campos y persiguieron a los simpatizantes del Nacionalismo en las agencias federales. El escalamiento de este conflicto tuvo una serie de efectos a nivel político global. En primer lugar, algunos sectores del Gobierno norteamericano sugirieron que debía otorgarse la independencia a Puerto Rico, no tanto para solucionar los problemas de esta isla, sino como una represalia por el asesinato de Riggs. Con el estado dependiente de la economía, una súbita independencia resultaba una verdadera amenaza para amplios sectores de la población isleña, cuya existencia misma dependía del sistema económico introducido por los Estados Unidos. El Proyecto Tydings de 1936, presentado al Congreso con el fin de liberar a los Estados Unidos de sus problemas en Puerto Rico, no tuvo mayor éxito. Sin embargo, tuvo un impacto de proporciones en la vida política de la isla. Muchos de los liberales, que habían favorecido la independencia antes de los sucesos de los años treinta, reaccionaron con alarma ante la posibilidad de una independencia. Un temor inconfesado ante la reacción de los Estados Unidos, dramatizada por la represión de los nacionalistas, parece haber hecho mella en la confianza de algunos liberales por conseguir la independencia. Para muchos, resultaba claro que el Gobierno de los Estados Unidos no estaba dispuesto a acceder a demandas bajo presion para solucionar el status de la isla, ni tampoco a abandonarla, como recientemente lo había demostrado el fracaso del Proyecto Tydings. Nuevas formulaciones políticas se hacían necesarias ${ }^{10}$.

\section{El Estado Libre Asociado}

EI arquitecto de la nueva política que empieza a dominar Puerto Rico en 1940, y que se mantiene hasta el presente, fue Luis Muñoz Marín. Hijo de Luis Muñoz Rivera, quien había sido Jefe del Gobierno en el breve lapso transcurrido entre la retirada de los españoles y la entrada de los norteamericanos, Muñoz Marín había apoyado en un principio las tesis independentistas. Los hechos de los. años treinta, sin embargo, le llevaron a replantear sus postulados políticos y establecer un nuevo tipo de relaciones con los Estados Unidos. En primer lugar, reconocer la dominación norteamericana, para después negociar un grado mayor de autonomía para Puer-

\footnotetext{
${ }^{10}$ Todos estos hechos se encuentran descritos por Diana Ghristophulos, "Puerto Rico in the Twentieth Century: A Historical Survey", en Petras y López, Puerto Rico, pp. 123-163. También en Maldonado-Denis, pp. 83-129 y en Lewis, pp. 123-142.
} 
to Rico, Esta fórmula, intentada ya en el siglo diecinueve bajo España, consiguió una vez más un apoyo político bastante amplio, como también el beneplácito de Washington. En 1938, Luis Muñoz Marín funda el Partido Popular Democrático (PPD), que se mantiene como fuerza política de importancia hasta el presente. Luis Muñoz Marín y el PPD lograron cambiar en alguna medida el grado de extrema dependencia política que predominó en Puerto Rico durante los primeros cuarenta años de intervención norteamericana. Sin embargo, como los críticos lo señalan, la autonomía lograda es función directa de un cambio de política en Washington.

De hecho, a un primer período de "negliggencia" norteamericana, como la denomina un autor, sucede un período ampliamente conocido como el New Deal, que se distinguió por una serie de reformas sociales y económicas durante las administraciones de Franklin Delano Roosevelt ${ }^{11}$. El impacto del New Deal resultaría de gran importancia para la vida política de la isla. Si bien en los primeros años de intervención los Estados Unidos se habían preocupado de la isla sólo en la medida en que favoreciera sus intereses comerciales, militares y estratégicos, esta situación cambiaría drásticamente durante la era Roosevelt. Antes de este período, los gobernadores designados por el Gobierno norteamericano no sólo carecían de la experiencia necesaria, sino que no conocían ni la tradición política ni el lenguaje de Puerto Rico. Estos puestos eran otorgados como recompensas por logros o desgracias políticas ocurridas en Washington. Para muchos gobernadores, el puesto en Puerto Rico representaba o un exilio político o una vacación tropical antes de la jubilación. Por muchos años, no había prácticamente comunicación entre puertorriqueños y norteamericanos en asuntos relativos al gobierno de la isla.

Pero a partir de la Administración de Roosevelt, la situación comienza a cambiar. El New Deal se hizo sentir rápidamente en Puerto Rico con la extensión de la ayuda federal que antes se otorgaba solamente a los estados. Pero por sobre todo se hizo sentir en la mayor comunicación entre los círculos politicos de la metrópolis y los políticos locales en la isla. También se hizo sentir a través de la designación de gobernadores más capaces, como el caso de Rexford Tugwell en $1941 \mathrm{y}$ la iniciación de un programa de reformas fundamentales en la isla a partir de entonces.

EI año 1940 representa un hito de suma importancia en la historia de Puerto Rico. Este es el año en que el PPD asume el control de la Legislatura y Luis Muñoz Marín es elegido presidente del Senado. Desde entonces hasta 1968, el PPD controlará el Gobierno de la isla en elecciones realizadas cada cuatro años y en las que consistentemente obtiene la mayoría de los votos. La victoria de 1940

IEste perfodo ha sido ampliamente descrito por Gordon Lewis en su obra, Puerto Rico. 
señala sólo el comienzo, puesto que en 1948, por primera vez, un puertorriqueño asume el cargo ejecutivo de mayor importancia en la isla: Luis Muñoz Marín es el primer Gobernador electo en Puerto Rico luego de una sucesión de gobernadores nombrados en Wash. ington.

Gon Luis Muñoz Marín comienza una era de profundas transformaciones económicas y políticas, aunque siempre bajo el alero de los Estados Unidos. En lo político, Muñoz Marín promulga el status político que define hasta nuestros días la situación política de Puerto Rico en relación a los Estados Unidos, y que proporciona también su identidad internacional. Se trata del "Estado Libre Asociado", aprobado en 1952 con el apoyo del 70 por ciento del electorado, que define a Puerto Rico como un estado relativamente independiente que goza de una relación especial con Ios Estados Unidos. En realidad, esta definición representa en gran medida un intermedio arbitrario entre extremos indeseables. $\mathrm{Ni}$ la independencia absoluta ni la incorporación total han resultado completamente deseables para la opinión pública puertorriqueña. Y tampoco para los Estados Unidos. Así, una solución intermedia, como la de un "Estado Libre Asociado", suministra una apariencia de solución para una situación extremadamente delicada. EI mismo MIuñoz Marín planteó esta situación en los siguientes términos, al describir los antecedentes políticos del Estado Libre Asociado:

Ambas abstracciones [la estadidad y la independencia] eran adversas a la solución de muchos otros problemas puertorriqueños. Así, pues, el dilema permanentemente planteado ante el pueblo equivalía a formularle la siguiente absurda pregunta: "¿Cuál es tu manera predilecta de impedir la solución de los problemas vitales de Puerto Rico?". Con la independencia desaparecería el mercado libre. Con la estadidad aparecería el cobrador de impuestos federales. Pero la premisa era de que, aparte de la independencia o la estadidad, sólo cabía la indignidad del colonialismo. ¿Qué escoges: comerte tu pan avergonzado o proclamar tu dignidad hambriento? La carga que ese dilema-que se suponía inexorable- hacía pensar sobre el alma de un pueblo bueno, digno y altivo, no es fácil de exagerar ${ }^{12}$.

En los términos de Muñoz Marín, la solución política debía no sólo adecuadarse sino subordinarse a las necesidades económicas. So-

\footnotetext{
IEI artículo al que pertenece este extracto fue originalmente publicado en la revista Foreign Affairs, en 1954. También se encuentra reimpreso bajo el nombre de "Puerto Rico, los Estados Unidos y el Estado Libre Asociado: por Luis Muñoz Marín, Gobernador del Estado Libre Asociado de Puerto Rico, Año 1954", en Eugenio Fernández Méndez, Crónicas de Puerto Rico, desde la Conquista hasta nuestros dias: 1493-1955 (Puerto Rico: Editorial Universitaria de Puerto Rico, 1976), pp. 651-666.
} 
Iván Jaksić / Puerto Rico: los dilemas del status político y el desarrollo...

bre la base de este supuesto, la administración de los populares, como se conoce comúnmente a los miembros del PPD, introdujo un cambio fundamental en la economía del país: el traslado de la economía agrícola a la economía industrial. En efecto, la economía basada en el azúcar, el café y el tabaco ya había hecho crisis durante los años treinta no sólo por la depresión, sino por las políticas de tarifas e impuestos introducidos por los Estados Unidos. El azúcar estaba sujeta a cuotas, mientras que el café y el tabaco eran adquiridos por los Estados Unidos en otros mercados del Caribe y de Latinoamérica. Al mismo tiempo, Puerto Rico, de acuerdo a las leyes en vigencia, no podía vender sus productos a otros mercados fuera de los Estados Unidos. En este sentido, Puerto Rico fue víctima no sólo de las leyes propias de la explotación comercial de la agricultura sino que también del sistema de contribuciones implantado por los Estados Unidos. De cualquier modo, este tipo de economía ya había hecho crisis en el momento en que los populares asumen el poder.

La agricultura, al menos hipotéticamente, podría haber sido desarrollada en otros términos que los del monocultivo, como por ejemplo enfatizando un desarrollo agrario más diversificado y con la capacidad de abastecer las necesidades alimenticias de la población. El patrón económico del Caribe, sin embargo, ha sido tradicionalmente el de producir un solo artículo para comprar a precios inflacionarios el resto necesario para la subsistencia. Este continuó siendo el patrón económico en Puerto Rico, aún bajo la dominación norteamericana. Pero en lugar de desarrollar otros aspectos de la agricultura, el PPD decidió desarrollar el país bajo un modelo de industrialización dependiente.

\section{EL desarrollo industrial y su impagto en Puerto Rico}

Inicialmente, la industrialización parecía suministrar la mejor fórmula de desarrollo en tanto no parecía haber otras alternativas viables para absorber el creciente desempleo derivado del quiebre de la economía agrícola. Más que un modelo de industrialización, el plan del PPD consistía en atraer capital norteamericano e industrias que a su vez crearan nuevas fuentes de trabajo. Obviamente, como no había compañía interesada en instalarse en Puerto Rico con el solo afán de desarrollar la isla, se dieron una serie de facilidades que si bien atrajeron a muchas firmas norteamericanas, también acentuaron la dependencia y produjeron pocos beneficios. En primer lugar, se estableció un sistema de exención de impuestos que permitía a las firmas instalarse por diez años sin tener que pagar impuestos. Una reforma de la Ley de Incentivos Económicos en 1963 extendió esta franquicia a 17 años. Pero la mayor atracción la suministraba la mano de obra barata en relación al precio 
de la misma en los Estados Unidos. Como garantía, el Gobierno de Puerto Rico legisló siempre en contra de la imposición del salario mínimo, de modo de atraer la inversión a Puerto Rico. Esto no hubiera sido posible sin la colaboración activa de los sindicatos en Puerto Rico. Muy al contrario de la militancia de los sindicatos en la mayor parte de América Latina, los sindicatos en Puerto Rico lucharon por sus logros dentro del contexto del desarrollo dependiente. En este contexto, la promesa de incrementar las fuentes de trabajo contenid.a $\epsilon$ el programa de desarrollo industrial les hizo apoyar las medidas del Gobierno para atraer el capital norteamericano. Sin un salario mínimo que imponérseles, y con una serie de franquicias en impuestos, muchas compañías norteamericanas comenzaron a instalarse en la isla.

Inicialmente, los resultados fueron impresionantes. Se estableció una maquinaria de gobierno que hizo bastante eficiente la administración del nuevo programa económico. La Administración para el Fomento Económico, más conocida como FoMENTo, fue creada en 1950 con tal propósito. Se crearon además oficinas de planificación, presupuesto y estadística que facilitaron la introducción masiva de industrias norteamericanas en la isla. En 1953, había cerca de 300 fábricas operando en Puerto Rico bajo el programa de industrialización conocido como "Operación Manos a la Obra". Estas industrias crearon 25.000 trabajos nuevos. El ingreso per cápita subió de 122 dólares en 1940 a 426 dólares en 1953. En 1960, habra 660 fábricas en funcionamiento, con 45.900 plazas nuevas. El costo del programa de industrialización entre 1950-1956 fue de 30 millones de dólares, mientras que las ganancias derivadas de este programa ascendieron a los 106 millones de dólares durante el mismo período ${ }^{13}$.

Estas cifras resultan impresionantes a primera vista, pero el crecimiento económico no implicó un desarrollo real para la isla. Por una parte, Puerto Rico se encontraba más dependiente que nunca del capital norteamericano. Por otra, el crecimiento de la población hacía las cifras de nuevas plazas de trabajo prácticamente irrelevantes. Además, el impacto de la industrialización tuvo una serie de otros efectos que aún requieren de examen ${ }^{14}$. Por ejemplo, la destrucción de la economía agrícola y el fracaso de la "Operación Manos a la Obra" por crear suficientes plazas, enviaron a miles de campesinos a las poblaciones callampas de San Juan y a las barriadas de Nueva York: 70.000 puertorriqueños emigraron a Nueva York en 1940. Esta cifra había aumentado a cerca de un millón hacia finales de la década de los sesenta, en circunstancias que la po-

${ }^{13}$ Lewis, pp. 170, 175.

14 Las limitaciones del programa de industrialización se encuentran descritas por Morris Morley en su artículo "Dependence and Development in Puerto Rico", en Petras y Lopez, Puerto Rico, pp. 214-254. 
blación total de la isla era de tres millones en ese momento.. El impacto de este desplazamiento masivo de puertorriqueños ha tenido consecuencias no sólo para la isla, sino también para los grandes centros urbanos de los Estados Unidos como Nueva York, Filadelfia y Chicago. El puertorriqueño constituye hoy un sector discriminado de la población norteamericana, aunque adquiere cada vez mayor influencia política ${ }^{15}$.

La emigración masiva, y el incremento de la dependencia son los precios que Puerto Rico hubo de pagar por su programa de industrialización. Este programa resultó exitoso en la medida en que creó nuevas fuentes de trabajo y reemplazó una estructura ya agónica de explotación comercial de la agricultura. Pero creó, en el aspecto negativo, una vulnerabiliclad muy grande respecto a los intereses del capital norteamericano. En efecto, el modelo sólo podía tener éxito en tiempos de expansión económica. Pero demostró ser altamente vulnerable en aquellas instancias en que las industrias decidieron cambiar sus centros de operaciones, o reemplazar la mano de obra por maquinaria. Por ejemplo, a partir de 1962, el promedio de trabajadores por fábrica disminuyó de 80 a $55^{16}$. Y sobre este tipo de problemas, el Gobierno de Puerto Rico no tuvo, ni tiene, mayor influencia.

Así, el exito del programa de industrialización resultó ser no sólo limitado, sino también de corta vida. Con el fracaso de este programa, la economía de Puerto Rico se hizo más dependiente de la ayuda federal de los Estados Unidos. Entre 1933 y 1946, por ejemplo, Puerto Rico recibió 230 millones de dólares en ayuda federal. Para 1972, esta cifra había ascendido a 390 millones de dólares, para ese año solamente ${ }^{17}$. Una economía basada en este tipo de subvenciones, tal como la basada en la industrialización, también depende de los periodos de expansión económica de los Estados Unidos. Es decir, Puerto Rico puede depender de la ayuda federal en tanto el flujo sea constante y no varíe de acuerdo a los cambios políticos en Washington, o los períodos de recesión económica. Durante los años sesenta, esta situación no parecía representar mayor problema, aunque hoy hace crisis. En efecto, los recientes cortes presupuestarios federales para una serie de programas de servicio social han afectado seriamente la economía de Puerto Rico. Es decir, la dependencia se ha institucionalizado en periodos de expansión,

"rVease Adalberto López, "The Puerto Rican Diaspora: A Survey", en Petras y López, Puerto Rico, pp. 316-146. La peregrinación migratoria desde las montañas del interior puertorriqueño a los arrabales neoyorquinos ha sido brillantemente narrada por René Marqués en su obra La carreta (Río Piedras, Puerto Rico: Editorial Gultural, I963). También en un plano literario, la vida de los puertorriqueños en Nueva York ha sido descrita por Pedro Juan Soto en Spiks, tercera edición (Rio Piedras: Editorial Gultural, 1970).

${ }^{10}$ Morris Morley, "Dependence and Development in Puerto Rico", p. 226.

${ }^{17}$ Ibid., p. 228 . 
pero su verdadera naturaleza se hace patente en periodos de crisis. El caso resulta particularmente dramático en el área educacional, que depende casi fundamentalmente de las subvenciones provenientes de Washington. De la misma manera, Ios servicios médicos y los subsidios de alimentación, de los cuales la población puertorriqueña ha debido depender debido al alto nivel de cesantía, amenazan gravemente la estabilidad política del país.

Aunque el foco principal de este estudio es la relación entre el status politico y el desarrollo económico dependiente, es necesario examinar, siquiera someramente, el impacto de la influencia norteamericana en otras áreas de la vida del país. Desde el comienzo de la intervención norteamericana, por ejemplo, se hicieron sentir los efectos de la erosión sistemática del catolicismo como religión dominante en la isla a través de la introducción del protestantismo, y los efectos de la introducción del inglés como lenguaje oficial en el sistema educacional. Si bien es cierto que la mayoría de los isleños no tenian mucho que añorar respecto a un pasado hispano que recordaba más que nada la pobreza, la esclavitud y la subordinación de siglos, no es menos cierto que el Ienguaje castellano y la religión católica suministraron algunos de los primeros focos de crítica y oposición a la intervención norteamericana. No resulta de sorprender que aquellos sectores más favorecidos durante la dominación española se transformaran en los críticos de la norteamericana. Así, la defensa del castellano y del catolicismo tuvo una inspiración elitista que, sin embargo, a lo largo de los años ha adquirido caracteres más populares y nacionalistas. El reemplazo del inglés por el castellano como el lenguaje oficial de la Universidad de Puerto Rico en 1948, por ejemplo, representa un hito de importancia en la historia cultural contemporánea de la isla ${ }^{18}$.

Las transformaciones más profundas de la sociedad puertorrique$\tilde{n} a, \sin$ embargo, trascienden el lenguaje y la religión. La intervención norteamericana trajo consigo una serie de divisiones sociales que alteraron profundamente los patrones tradicionales de vida comunitaria y familiar. El surgimiento de clases vitalmente conectadas con la actividad norteamericana en la isla, como por ejemplo una nueva clase media concentrada en las áreas del comercio y la administración, además de una clase trabajadora surgida de la nueva economía industrial, rompieron tal vez para siempre la organización social compuesta por una élite peninsular y una población. fundamentalmente campesina que es característica de la domina-

${ }^{28}$ Sobre la actividad estudiantil y el foco de oposición universitaria en Puerto Rico, puede consultarse Isabel Picó, "Origins of the Puerto Rican University Student Movement Undex v.s. Domination (1903-1930)", en Petras y López, Puerto Rico, pp. 175-194. También Arthur Liebman, The Politics of Puerto Rican University Students (Austin and London: The University of Texas Press, 1970). 
ción española. En el plano familiar, la entrada de la mano de obra femenina en las nuevas industrias alteró profundamente los marcos patriarcales que caracterizaban a la familia bajo el sistema económico español. Estos cambios, si bien coinciden con la intervención norteamericana en Puerto Rico, no se restringen ni a uno ni a otro pafs, sino que resultan propios de la industrialización y la modernización en otros puntos del planeta. Pero de cualquier modo, los cambios en Puerto Rico fueron drásticos y profundos.

Es en el plano del consumo donde los cambios operados en la sociedad puertorriqueña resultan más ostensibles. La apertura del mercado puertorriqueño para los productos norteamericanos trajo consigo la introducción masiva de productos característicos del consumo norteamericano. En los comienzos del programa de industrialización, por ejemplo, cuando Puerto Rico tenía sólo dos millones de habitantes, la isla ya se habia transformado en el segundo comprador más importante en América Latina, y el noveno en el mundo, de productos norteamericanos ${ }^{19}$. Puerto Rico, debido a su relación especial con los Estados Unidos, fue uno de los primeros pafses subdesarrollados en experimentar un fenómeno que hoy resulta característico de los países que reciben los artículos de consumo normal en países ya desarrollados: el producto en cuestión adquiere un valor más simbólico que práctico. Así, la ciudad de San Juan, que por sus características urbanas no se encuentra preparada para tolerar los millares de automóviles que hoy la recorren, paga el precio de una adquisición inorgánica de nuevos patrones de consumo. Un crítico señala cómo los alimentos envasados adquirieron súbitamente el carácter de símbolos de status social, en circunstancias que abundan en el país una serie de envidiables productos tropicales frescos. El mismo crítico señala cómo el televisor fue el único producto capaz, en muchos hogares, de erradicar al refrigerador del living-room, en donde había sido instalado para hacer resaltar el status alcanzado bajo el nuevo orden económico ${ }^{20}$. Este tipo de consumo tecnológico inorgánico respecto a la sociedad consumidora ha generado una serie de transtornos culturales que llevan a muchos a buscar en el pasado hispano un sustento para enfrentar el cambio acelerado. Tales transtornos resultan propios de una sociedad en rápido, aunque desordenado, desarrollo económico. Con la crisis de este desarrollo, los problemas que afectan a Puerto Rico van más allá de lo político y económico, para afectar también lo social y cultural.

\section{LA GRISIS DEL STATU QUO POLfTICO Y ECONÓMICO}

A pesar de la gravedad de los problemas de Puerto Rico, no exis-

${ }^{18}$ Petras y López, p. 106.

${ }^{\infty}$ Lewis, p. 243. 
ten los mecanismos internos en el sistema que permitan su solución. La creación de tales mecanismos implicaría necesariamente un replanteamiento del status político de la isla. Es decir, sólo como país soberano e independiente podría Puerto Rico, hipotéticamente, tomar las decisiones necesarias para cambiar drásticamente la dirección de la economía. El status político actual, en este sentido, se encuentra íntimamente ligado a la economía dependiente. Pero dadas las condiciones estratégicas y militares bajo las cuales se encuentra Puerto Rico en el ajedrez estratégico mundial, no resulta prácticamente posible plantear un programa independentista sin suscitar la ira de los Estados Unidos. El Departamento de Estado norteamericano, además, ha declarado explícitamente que "el status político de la isla debe ser congruente con los legitimos requerimientos del Departamento de Defensa en el área del 'Garibe"2I. El independentismo, en este contexto, no representa sino una amenaza para las numerosas bases militares instaladas en Puerto Rico, algunas de las cuales están equipadas con armas nucleares. El bloqueo de Cuba, por otra parte, habla elocuentemente sobre las posibilidades del independentismo. Sin embargo, la continuación del statu quo conlleva un agravamiento de la crisis. Desde 1952 en adelante, Puerto Rico pudo desarrollarse bajo las guías del crecimiento dependiente gracias a que la economía misma de los Estados Unidos se encontraba en un período de expansión. Pero dadas las circunstancias actuales de recesión, la crisis del modelo económico conlleva naturalmente la crisis del modelo político. La definición de Estado Libre Asociado suministró originalmente un aplazamiento del conflicto entre independencia y estadidad, pero la crisis actual lo resucita. El Estado Libre Asociado, además, proporcionó en su momento de origen, ejemplos tangibles de crecimiento económico. Pero al surgir la actual crisis económica, la prolongación del statu quo que genera sólo mayores aprehensiones y el agravamiento de la situación de dependencia.

Una primera muestra de la crisis a nivel político fue suministrada por la derrota del PPD en las elecciones de 1968. Hasta poco antes de esa fecha, el apoyo al statu quo era mayoritario, como se ve reflejado en los resultados del plebiscito de 1967 relativo al status político. En ese plebiscito, el Estado Libre Asociado recibió un 60.4 por ciento de los votos, la estadidad un 38.9 por ciento y el independentismo un 0.6 por ciento ${ }^{22}$. Pero en las elecciones de I968, como reflejo de una escisión en el PPD, pero también como reflejo de un descontento con el statu quo, los partidarios de la estadidad obtuvieron la gobernación de la isla por primera vez en la historia

"Senior Seminar in Foreign Policy, "The Problem of Puerto Rico's Political Status", Seventeenth Session, Department of State 1974-1975

"Aaron Gamaliel Ramos, "The Development of Annexationist. Politics in Twentieth Century Puerto Rico", en López, The Puerto Ricans, p. 266. 
de la intervención norteamericana. Aunque los populares retornaron al poder en 1972, perdieron nuevamente en las elecciones de 1976 ante las fuerzas pro-estadistas del Partido Nuevo Progresista (PNP), que se encuentra actualmente en el poder. Contrariamente a lo que amplios sectores esperaban de la victoria de los pro-estadistas, la toma del poder no ha significado la obtención del status de estado norteamericano para Puerto Rico. Esta situación ha dejado en claro que incluso la supuesta autonomía política de Puerto Rico tiene límites muy definidos en lo que respecta a la estadidad. Tanto las consideraciones económicas como las estratégicas han hecho que los Estados Unidos continúen apoyando el actual Estado Libre Asociado, a pesar de sus obvias limitaciones económicas.

\section{CoNCLUSIóN}

La situación actual de Puerto Rico encierra un dilema fundamental: no puede resolverse la actual crisis económica, y otras futuras que resultan propias del desarrollo dependiente, sin un cambio fundamental en el status político. Sin embargo, el cambio en el status político resulta no sólo intolerable para los Estados Unidos, sino también para amplios sectores de la sociedad puertorriqueña que han llegado a depender vitalmente de una economía sustentada por el capital privado y la ayruda federal de los Estados Unidos. La raíz de los problemas actuales radica no tanto en que los Estados Unidos se encuentren en un periodo de recesión económica que eventualmente logrará ser superado, sino en el fundamento mismo del desarrollo dependiente. La base política de Puerto Rico, inspirada en el esquema de Estado Libre Ásociado en relación a los Estados Unidos, institucionalizó los mecanismos de dependencia económica que sólo podían ser legitimados mediante una solución intermedia entre independencia y estadidad. Una alteración del status económico, de este modo, requiere una alteración del status político. Pero no hay evidencia que tal status político será alterado hasta que el modelo alcance las proporciones de una crisis total. 